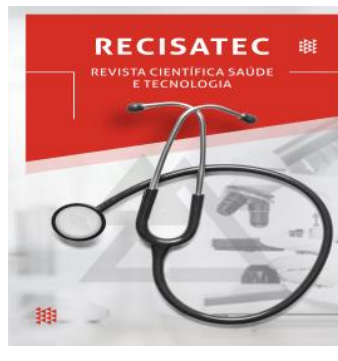

RECISATEC - REVISTA CIENTÍFICA SAÚDE E TECNOLOGIA ISSN 2763-8405

\title{
BENEFÍCIOS DA ATIVIDADE FUNCIONAL EM IDOSOS COM DOENÇA DE PARKINSON: REVISÃO BIBLIOGRÁFICA
}

\section{BENEFITS OF FUNCTIONAL ACTIVITY IN ELDERLY WITH PARKINSON'S DISEASE: BIBLIOGRAPHIC REVIEW}

Kauara Vilarinho ${ }^{1}$, Ane Beatriz Vital de Castro², Alana Freitas Santos ${ }^{3}$

Submetido em: 16/09/2021

Aprovado em: 26/10/2021 e1433

https://doi.org/10.53612/recisatec.v1i4.33

\begin{abstract}
RESUMO
O paciente com Doença de Parkinson (DP) apresenta disfunções ou distúrbios motores tais como tremores de repouso, rigidez muscular, lentidão do movimento, além de acometer a marcha e o padrão postural. O comprometimento físico-mental associado com os sinais e sintomas e aos distúrbios secundários da DP podem resultar num agravamento considerável da qualidade de vida (QV), o que pode levar o indivíduo portador dessa condição ao isolamento social. Portanto, o objetivo desse trabalho foi verificar a importância da fisioterapia, através de atividades funcionais, na melhora da parte motora desses pacientes. Para tanto, pesquisou-se artigos entre os anos de 2011-2021 nas bases de dados LILACS, Google acadêmico e Bireme com os descritores doença de Parkinson, fisioterapia, atividade funcional, treino de equilíbrio. O estudo concluiu que a fisioterapia tem um importante papel na reabilitação e redução dos limites funcionais do paciente com DP, promovendo assim uma melhor independência funcional, o que poderá gerar uma melhor qualidade de vida para os mesmos.
\end{abstract}

PALAVRAS-CHAVE: Doença de Parkinson. Fisioterapia. Atividade funcional. Treinamento de equilibrio

\begin{abstract}
The patient with Parkinson's Disease (PD) presents dysfunctions or motor disorders such as resting tremors, muscle rigidity, slowness of movement, in addition to affecting the gait and postural pattern. The physical-mental impairment associated with the signs and symptoms and secondary disorders of $P D$ can result in a considerable worsening of the quality of life (QL), which can lead the individual with this condition to social isolation. Therefore, the objective of this study was to verify the importance of physical therapy, through functional activities, in the improvement of the motor part of these patients. For that, articles were searched between the years 2011-2021 in the LILACS, Academic Google and Bireme databases with the descriptors Parkinson's disease, physiotherapy, functional activity, balance training. The study concluded that physiotherapy has an important role in the rehabilitation and reduction of the functional limits of patients with $P D$, thus promoting better functional independence, which will generate a better quality of life for them.
\end{abstract}

KEYWORDS: Parkinson's disease. Physiotherapy. Functional activity. Balance training.

\section{INTRODUÇÃO}

A doença de Parkinson (DP) é caracterizada como uma doença neurológica crônica, degenerativa e progressiva, que acomete o sistema motor. A DP se desenvolve quando há uma perda

\footnotetext{
1 Faculdade Santa Inês - UNIBRAS

2 Faculdade Santa Inês - UNIBRAS

3 Faculdade Santa Inês - UNIBRAS 


\section{RECISATEC - REVISTA CIENTÍFICA SAÚDE E TECNOLOGIA} ISSN 2763-8405

BENEFÍCIOS DA ATIVIDADE FUNCIONAL EM IDOSOS COM DOENÇA DE PARKINSON: REVISÃO BIBLIOGRÁFICA Kauara Vilarinho, Ane Beatriz Vital de Castro, Alana Freitas Santos

rápida dos neurônios do Sistema nervoso Central (SNC), localizados no mesencéfalo, na região conhecida como substância negra, responsável pela produção e liberação da dopamina, um neurotransmissor, cuja sua função envolve controle dos movimentos. ${ }^{1}$

Segundo bases de 2017 da Organização Mundial da Saúde (OMS), a DP acomete mundialmente $1 \%$ da população, com idade acima de 65 anos e do sexo masculino. A patologia tem uma prevalência, segundo estatísticas disponíveis, de 150 a 200 casos a cada 100 mil habitantes e a cada ano ocorre uma incidência de 20 casos a cada 100 mil habitantes. ${ }^{2,3}$

As alterações da doença de Parkinson são várias, entre elas destaca-se, o déficit de equilíbrio e a falta de coordenação motora. Essas desordens motoras, se não tratadas a tempo, podem levar o idoso ao isolamento social, perda de vontade para as atividades que antes costumava fazer, dependência para as atividades de vida diária, perda de autonomia e consequentemente redução de sua qualidade de vida. ${ }^{4}$

Clinicamente a DP é caracterizada por sintomas motores. Por ser uma doença debilitante, progressivamente esses sintomas geram limitações significativas na função motora, instabilidade postural e equilíbrio que contribuem para a piora do seu bem-estar e suas tarefas funcionais. Intervenções Fisioterapêuticas com programas de exercícios para a DP, consistem num meio eficaz para a prevenção de quedas e independência diária, sendo diversas as formas de reabilitação que buscam a funcionalidade dos pacientes com DP. 5,6

Diante da necessidade de promover maior autonomia e independência surgiu uma modalidade de exercício físico, o treinamento funcional. Esse treinamento é feito baseado na funcionalidade, o qual preconiza a realização de movimentos integrados e multiplanares simulando aos movimentos feito nas atividades de vida diária. Os movimentos devem ser acompanhados de alguns elementos como a aceleração, estabilização e desaceleração, com o objetivo de aprimorar as habilidades de movimento, força do tronco e eficiência neuromuscular. ${ }^{7}$

As atividades funcionais são aquelas que objetivam o aprimoramento equilibrado das capacidades biomotoras no intuito de garantir eficiência e segurança para o desempenho das atividades cotidianas e/ou esportivas, respeitando bases teóricas e científicas e adequando-se às necessidades individuais do praticante. Em outras palavras, é composta por um programa de exercícios cuja finalidade é a melhora na capacidade funcional, estimulando o corpo humano de maneira a adaptá-lo as atividades cotidianas, aos esportes e reabilitação de lesões. ${ }^{8}$

As perdas funcionais progressivas que a população idosa enfrenta com o avançar da idade, causam importantes déficits, que com o tempo se tornam mais significativos e no intuito de minimizálos, as Atividades funcionais, trazem, no que diz respeito ao equilíbrio e coordenação motora de idosos com Parkinson, inúmeros benefícios, proporcionado ganho de propriocepção, força, resistência, flexibilidade, e condicionamento cardiovascular. Assim como auxilia na obtenção de funcionalidade, contribuindo para a manutenção da independência e qualidade de vida. ${ }^{9}$ 
RECISATEC - REVISTA CIENTÍFICA SAÚDE E TECNOLOGIA ISSN 2763-8405

BENEFÍCIOS DA ATIVIDADE FUNCIONAL EM IDOSOS COM DOENÇA DE PARKINSON: REVISÃO BIBLIOGRÁFICA Kauara Vilarinho, Ane Beatriz Vital de Castro, Alana Freitas Santos

Este trabalho tem por objetivo analisar a importância da fisioterapia, através das atividades funcionais, em promover a manutenção do equilíbrio e coordenação motora em paciente acometidos pela doença de Parkinson, preservando assim sua autonomia funcional.

\section{DOENÇA DE PARKINSON: CONCEITO E FISIOPATOLOGIA}

A doença de Parkinson (DP) é uma desorganização crônica, progressiva e polissintomática do sistema nervoso central, associada a disfunções do movimento, que pode provocar alterações cognitivas, comportamentais e de humor, além da demência que, ocorre em $25 \%$ dos indivíduos afetados. Estas alterações iniciam de forma gradual e lenta e tendem a agravar com o tempo. ${ }^{10}$

O Parkinson é uma doença crônica degenerativa, progressiva que ataca o Sistema Nervoso Central e provoca morte dos neurônios dopaminérgicos, células que produzem a dopamina, importante neurotransmissor que atua na execução dos movimentos, e se localizam na região negra do cérebro. ${ }^{11}$

De acordo com alguns estudos, esta enfermidade degenerativa cursa com uma perda progressiva de células da substância negra do mesencéfalo. A degeneração de neurônios da zona compacta da substância negra resultará numa diminuição da produção de dopamina, com disfunção da via nigroestriatal e subsequente perda da dopamina estriatal. Quando aproximadamente $80 \%$ dos neurônios da substância negra forem perdidos, a DP torna-se evidente e os indivíduos começam a ter grandes dificuldades. ${ }^{12}$

Estudos revelam que a incidência da DP é de aproximadamente 85 a 187 casos por 100.000 pessoas, ou seja, $1 \%$ da população mundial. Este distúrbio acomete principalmente pessoas idosas, na faixa etária entre 55 e 65 anos com maior prevalência em pessoas do sexo masculino. Porém, em alguns anos, o início da doença pode ocorrer mais precoce, antes de 40 anos ou até mesmo abaixo dos 21 anos de idade, quando isso ocorre dá-se o nome de Parkisionismo precoce. ${ }^{13}$

A doença de Parkinson fisiopatologicamente deve ser considerada como uma afecção neurodegenerativa, progressiva, caracterizada pela presença de disfunções monoaminérgicas múltiplas, incluindo déficits dos sistemas dopaminérgicos, colinérgicos, serotoninérgicos e noroadrenérgicos. ${ }^{14}$

\section{PRINCIPAIS ALTERAÇÕES FUNCIONAIS}

Pacientes com doença de Parkinson, apresentam redução significativa das fibras musculares do tipo IIA, o que gera a atrofia por desuso ou descondicionamento físico e predispõe à fraqueza e fadiga muscular. Os pacientes com DP também possuem uma rigidez muscular global. A redução de massa muscular leva à incoordenação de movimentos, quedas frequentes, intolerância ao exercício e piora progressiva do condicionamento físico, fato que limita as atividades de vida diária. ${ }^{15}$

Esta patologia é caracterizada pela apresentação de distúrbios motores progressivos, como o tremor de repouso, bradicinesia, rigidez, alterações de postura e marcha. As manifestações clínicas apresentam-se inicialmente com predomínio unilateral, progridem para manifestações bilaterais e em 


\section{RECISATEC - REVISTA CIENTÍFICA SAÚDE E TECNOLOGIA} ISSN 2763-8405

BENEFíCIOS DA ATIVIDADE FUNCIONAL EM IDOSOS COM DOENÇA DE PARKINSON: REVISÃO BIBLIOGRÁFICA Kauara Vilarinho, Ane Beatriz Vital de Castro, Alana Freitas Santos

estágios mais avançados causam a perda da independência funcional. Outros distúrbios encontrados em indivíduos com a Doença de Parkinson são anormalidades nas aferências sensoriais e no processamento motor sensorial. ${ }^{16}$

A doença de Parkinson afeta e prejudica as estruturas e as funções do corpo, causando limitações nas atividades de vida diária. A fisioterapia voltada para a DP tem como objetivo minimizar os problemas motores, ajudando o paciente a manter a independência para realizar as atividades de vida diária. Com o exercício, o aumento da mobilidade pode de fato modificar a progressão da doença e impedir contraturas, além de ajudar a retardar a demência. ${ }^{12}$

A doença de Parkinson leva a uma condição de hipocinesia, incluindo: Perda de força muscular, lentidão de movimento (bradcinesia), dificuldade no ato motor (acinesia), hipertonia muscular (rigidez), instabilidade postural, tremor das mãos e mandíbula. Tais sintomas originam-se da neurodegeneração dos neurônios dopaminérgicos da substância negra e atenuação da dopamina. ${ }^{17}$

Para os portadores da DP, a alteração postural (AP) também é uma alteração frequente da doença de Parkinson (DP). Casos de maior comprometimento clínico, representados pela síndrome da cabeça caída (dropped head), pela camptocormia e pela síndrome de Pisa, são exemplos de posturas anormais graves que podem acometer os pacientes portadores da DP. A prevalência destas alterações na DP varia de 3 a $18 \%$, de acordo com os estudos realizados. ${ }^{18}$

\section{ATIVIDADES FUNCIONAIS}

As atividades que contemple habilidades motoras como equilíbrio, coordenação, marcha, agilidade e propriocepção, podem ser classificadas como atividades funcionais. As atividades funcionais são caracterizadas pela semelhança do exercício às necessidades e situações cotidianas, baseando-se na especificidade. Tem sido recomendado que o idoso pratique atividade física como forma de manter sua funcionalidade. ${ }^{19}$

Dentre as atividades mais praticadas, encontram-se a ginástica, caminhada, hidroginástica, dança, dentre outras. Entretanto, na atualidade, uma das alternativas para atingir melhorias na funcionalidade é a utilização das atividades funcionais considerando que a atividade funcional pode acarretar inúmeros benefícios para a população idosa e portadora de Parkinson, como: aumento da força muscular, potência muscular, melhora da capacidade cardiorrespiratória, equilíbrio, flexibilidade e cognição. ${ }^{20,19}$

As atividades funcionais objetivam o desenvolvimento integrado e equilibrado das diferentes capacidades físicas treináveis. Para isso, a atividade funcional utiliza exercícios multiarticulares e multiplanares, combinados a movimentos de aceleração, redução e estabilização, que tem como objetivo principal aprimorar a qualidade de movimento, melhorar a força da região da central do corpo e a eficiência neuromuscular para as necessidades específicas do idoso. ${ }^{20}$

A dupla tarefa ou atividade funcional, como também é chamada, pode ser definida como o ato de realizar uma atividade primária, para a qual é destinado o maior foco da atenção, incorporada a uma segunda atividade executada ao mesmo tempo. A realização de duas tarefas simultâneas é comum no 


\section{RECISATEC - REVISTA CIENTÍFICA SAÚDE E TECNOLOGIA} ISSN 2763-8405

BENEFÍCIOS DA ATIVIDADE FUNCIONAL EM IDOSOS COM DOENÇA DE PARKINSON: REVISÃO BIBLIOGRÁFICA Kauara Vilarinho, Ane Beatriz Vital de Castro, Alana Freitas Santos

cotidiano, e representa uma capacidade altamente vantajosa para o indivíduo, podendo ser considerada um pré-requisito para uma vida normal. ${ }^{21}$

Todas as atividades, sem exceção, devem ter por objetivo o desenvolvimento de alguma variável de funcionalidade. A prescrição de atividade funcional deve fornecer a adequada "dose" de exercícios frente às possibilidades de resposta ao estimulo e garantir adaptações ótimas em relação aos critérios de eficácia e funcionalidade. ${ }^{6}$

\section{TREINO DE EQUILÍBRIO E COORDENAÇÃO MOTORA}

O equilíbrio é um processo complexo que depende da integração da visão, da sensação vestibular e periférica, dos comandos centrais e das respostas neuromusculares e, particularmente, da força muscular e do tempo de reação. Para compreender o equilíbrio, é de extrema importância observar o sistema de controle postural e sua participação nas diversas tarefas motoras. ${ }^{11}$

Alguns autores relatam que a coordenação, é a interação harmoniosa e econômica do sistema musculoesquelético, do sistema nervoso e do sistema sensorial com o fim de produzir ações motoras precisas e equilibradas; e, reações adaptadas a situações que exigem adequada medida de força que determina a amplitude e a velocidade do movimento, uma apropriada seleção dos músculos que influenciam a condução e orientação do movimento e a capacidade de alternar rapidamente entre tensão e relaxamento muscular. ${ }^{22}$

O equilíbrio e a coordenação motora, são trabalhados através de exercícios de alongamento muscular, treino de equilíbrio, fortalecimento muscular, exercícios metabólicos e treino de força de membros inferiores, o que proporciona melhora do equilíbrio e também da marcha de idosos. Outro recurso terapêutico que tem se mostrado eficaz no equilíbrio e coordenação de idosos é a hidrocinesioterapia que consiste na aplicação de exercícios físicos com auxílio das propriedades da água. ${ }^{23}$

O equilíbrio corporal é a capacidade do ser humano de manter-se ereto ou executar movimentos de aceleração e rotação do corpo sem oscilação ou queda. A manutenção da postura é garantida pela interação sensório-motora, e a informação relevante, relacionada ao equilíbrio corporal, depende dos sistemas visual, somatossensorial e vestibular. ${ }^{24}$

Coordenação motora é à capacidade do corpo de realizar vários movimentos articulados e é resultado da interação entre os sistemas nervoso, muscular, esquelético e sensorial. Devido a ela que o ser humano e capaz de correr, andar, escrever, pintar, pular e desenhar. A coordenação motora entende, nos vários domínios, as alterações físicas, tais como perda de massa muscular e mudanças da estrutura esquelética; alterações motoras decrescentes de habilidades, como, por exemplo, força, equilíbrio, flexibilidade e alterações cognitivas, perda de memória e da capacidade de raciocínio rápido. ${ }^{25}$

Deve ser realizado um protocolo de exercícios de flexibilidade, força muscular, que de ênfase no tratamento do equilíbrio e coordenação motora e que melhore a capacidade física e o equilíbrio estático 
RECISATEC - REVISTA CIENTÍFICA SAÚDE E TECNOLOGIA ISSN 2763-8405

BENEFÍCIOS DA ATIVIDADE FUNCIONAL EM IDOSOS COM DOENÇA DE PARKINSON: REVISÃO BIBLIOGRÁFICA Kauara Vilarinho, Ane Beatriz Vital de Castro, Alana Freitas Santos

e dinâmico de idosos. Apesar do fortalecimento muscular ser indicado e apresentar evidências de melhora da força e potência muscular, exercícios funcionais também devem ser realizados visando à melhora do equilíbrio e coordenação e independência do idoso. ${ }^{26}$

\section{IMPORTÂNCIA DO TREINO DE EQUILÍBRIO E COORDENAÇÃO MOTORA NA DOENÇA DE PARKINSON}

A coordenação motora e equilíbrio com a evolução da doença, ficam comprometidos, o que faz com que o indivíduo diminua suas atividades diárias desencadeando uma atrofia muscular. A reeducação postural e a manutenção da atividade física são os principais focos da atuação fisioterapêutica no tratamento da DP. Os exercícios realizados visam à manutenção da atividade muscular e flexibilidade. ${ }^{27}$

A fisioterapia busca minimizar os problemas motores, ajudando o paciente a manter a independência para realizar as atividades de vida diária e melhorando sua qualidade de vida, exercício, o aumento da mobilidade pode de fato modificar a progressão da doença e impedir contraturas, além de ajudar a retardar a demência. ${ }^{12}$

Para trabalhar o equilíbrio, podem ser trabalhados: a cama elástica, onde o paciente realiza saltos em posição bipodal e unipodal, apoiado com os MMSS nas barras paralelas, e com auxílio do terapeuta caso necessário em duas séries de 10 repetições cada. Para o mesmo objetivo pode-se utilizar a prancha ortostática, em que o paciente permanece por no mínimo 5 minutos tentando manter o equilíbrio em cima da prancha, e sem apoio. Jogos dinâmicos em grupo, como arremessos de bolas ao cesto trabalha também o equilíbrio. ${ }^{28}$

As técnicas que trabalham a coordenação motora devem compreender, exercícios motores, treinamento de marcha, treinamento das atividades diárias. Os materiais utilizados consistiram em bolas suíças de todos os tamanhos, além de bolas esportivas (futebol, basquete e voleibol, cada qual com seu peso específico), tábuas de equilíbrio, bastões, e fitas adesivas. Além disso, a terapia com estímulos visuais, auditivo, os estímulos facilitariam os movimentos, o início e continuação da marcha, o aumento do tamanho dos passos e a redução da frequência e intensidade dos congelamentos. ${ }^{29}$

A fisioterapia promove benefícios na doença de Parkinson, ao englobar a orientação e prática de exercícios terapêuticos de alongamento, fortalecimento muscular, marcha, mobilidade, equilíbrio, transferência, relaxamento e exercícios respiratórios. ${ }^{30}$

Para atingir os objetivos em que o foco do tratamento fisioterapêutico e o equilíbrio, vários recursos são utilizados, entre eles a bola suíça, por ser um instrumento útil para avaliar e tratar problemas de equilíbrio e também da marcha, devido à necessidade de feedback vestibular e proprioceptivo para obter as respostas adaptativas apropriadas. ${ }^{31}$

Já a hidroterapia através das técnicas de Halliwick e Aí-chi são condutas bastantes viáveis para indivíduos com DP, pois auxiliam no tratamento para melhora da coordenação motora, e são técnicas que visam proporcionar independência funcional aos indivíduos com DP. Utilizam-se de movimentos leves e com vasta amplitude de movimento do corpo associado com a respiração, 


\section{RECISATEC - REVISTA CIENTÍFICA SAÚDE E TECNOLOGIA} ISSN 2763-8405

BENEFÍCIOS DA ATIVIDADE FUNCIONAL EM IDOSOS COM DOENÇA DE PARKINSON: REVISÃO BIBLIOGRÁFICA Kauara Vilarinho, Ane Beatriz Vital de Castro, Alana Freitas Santos

proporcionando o aumento da flexibilidade articular e muscular, através do estímulo sensorial corporal em todas as direções do fluxo da água. ${ }^{32}$

O treino de equilíbrio trabalhado através da cinesioterapia mostra-se fundamental para a melhora significativa no equilíbrio do idoso, e a melhoria em todas as variáveis que tiveram como desfecho o déficit de equilíbrio. Sua principal finalidade é a manutenção ou desenvolvimento livre para a sua função, e tem como efeitos principais a melhora da força, resistência a fadiga, coordenação motora, mobilidade e flexibilidade. ${ }^{33}$

O treinamento em esteira com ou sem suporte do peso corporal (no mínimo 20 minutos, duas a três vezes por semana, com aumento progressivo da carga, por pelo menos 6 semanas) é uma intervenção que melhora os desfechos da coordenação motora e também da marcha em pacientes idosos com doença de Parkinson. ${ }^{34}$

\section{ATIVIDADES FUNCIONAIS NO PARKINSON}

As atividades funcionais são um conjunto de exercícios com movimentos que imitam situações reais, ou seja, que podem ocorrer nas atividades da vida diária. Partindo de exercícios de estabilização e fortalecimento da musculatura que sustenta e estabiliza a coluna vertebral em cadeia com os membros, conhecido como musculatura do núcleo. E sua essência está baseada na melhoria dos aspectos neurológicos que afetam a capacidade funcional. ${ }^{35}$

As atividades funcionais são aprendidas durante toda a vida. Altamente vantajosa, é um prérequisito no desempenho funcional nas diversas atividades de vida diária. É de extrema importância para pacientes com Parkinson. Uma caminhada, por exemplo, permite a comunicação entre pessoas, atender um celular, transportar objeto de um local para outro, entre outras. Em circunstâncias normais a realização simultânea das tarefas cognitiva e motora é desempenhada automaticamente, enquanto, quando se apresenta uma alteração cognitiva e/ou no controle motor requer uma demanda atencional maior. ${ }^{36}$

Atividades funcionais devem serem feitas nos pacientes com Parkinson, da seguinte forma: de baixa velocidade para alta; posturas estáticas para dinâmicas; de menor intensidade para maior intensidade; movimentos controlados e conscientes para movimentos inconscientes e sem controle; de olhos abertos para fechados; movimentos bilaterais para unilaterais; movimentos simples para complexos; exercícios que exigem pouca coordenação para exercícios complexos; movimentos estáveis para movimentos instáveis; movimentos em um plano para movimentos em vários planos. ${ }^{37}$

A dupla tarefa ou atividade funcional, traz benefícios na marcha (melhorias na marcha curta, aumento do comprimento da passada e otimização da velocidade da marcha), na capacidade de dividir a atenção e até na fala, ao atenuar os sintomas de disartria. Além disso, o treinamento de situações de dupla tarefa trouxe benefícios no desempenho da área cognitiva. Os efeitos do exercício físico de dupla tarefa exercem efeitos positivos comportamentais e funcionais na qualidade de vida de pacientes com doença de Parkinson. ${ }^{38,39}$ 


\section{RECISATEC - REVISTA CIENTÍFICA SAÚDE E TECNOLOGIA} ISSN 2763-8405

BENEFÍCIOS DA ATIVIDADE FUNCIONAL EM IDOSOS COM DOENÇA DE PARKINSON: REVISÃO BIBLIOGRÁFICA Kauara Vilarinho, Ane Beatriz Vital de Castro, Alana Freitas Santos

A atividade funcional ou dupla tarefa se baseia na realização de duas tarefas motoras ou uma tarefa motora e uma cognitiva, e em muitas atividades diárias é altamente vantajoso executar mais de uma tarefa ao mesmo tempo. Em circunstâncias normais, quando há realização concomitante de tarefas motoras e cognitivas, as atividades motoras são desempenhadas de forma automática, pois não requerem recursos atencionais conscientes. ${ }^{40}$

Atividades funcionais envolvendo o treinamento cognitivo e de marcha, melhora a velocidade, o comprimento da passada e o tempo de apoio duplo em caminhada de dupla tarefa e caminhada simples em paciente com DP, com redução na variabilidade da marcha durante a caminhada. Portanto, demonstrou-se que o treino das atividades funcionais melhora o desempenho da marcha, do equilíbrio e coordenação motora desses pacientes, bem como melhora na QV. ${ }^{41}$

Exercício físico regular junto com as atividades funcionais, especialmente o aeróbico, traz muitos benefícios para indivíduos portadores de Parkinson, pois promove melhora da resistência muscular, melhora da marcha, melhora do equilíbrio e postura corporal, promove plasticidade neural, melhora da coordenação motora e da função cardiorrespiratória e em alguns casos, aumento na quantidade de neurônios, maior eficiência dos neurotransmissores e melhor captação de dopamina, tornando o exercício físico como uma importante ferramenta auxiliar ao tratamento desses indivíduos. ${ }^{1}$

\section{CONSIDERAÇÕES FINAIS}

Com base nos estudos analisados, foi demonstrado que a fisioterapia, através das atividades funcionais junto com o treino de equilíbrio e coordenação motora em idosos portadores da doença de Parkinson, apresentou um resultado positivo na vida destes idosos, aumentando seu grau de funcionalidade, deixando-os mais ativos, mantendo sua independência, trazendo inúmeros benefícios para sua vida. Entretanto é necessário que os idosos com DP continuem a praticar essas atividades funcionais seguidas de treino de equilíbrio e coordenação motora, não somente como tratamento, mais sim como uma forma de prevenção, mantendo, melhorando e prolongando a vida desses idosos.

\section{REFERÊNCIAS}

[1] Silva EM, Silva MW, Silva OAC, Silva MMJ, Silva SCG, Silva BRE, et al. Doença de Parkinson, exercício físico e qualidade de vida: uma revisão. Brazilian Journal of Development. 2020;6(9)1478-88.

[2] Organização Mundial de Saúde. Pacientes com Parkinson contarão com novos medicamentos no SUS. [acesso em: 2018 jun. 17]. Disponível em: http://portalms.saude.gov.br/noticias/agenciasaude/41873pacientescom-parkinson-contarao-com-novos-medicamentos-no-sus

[3] Queiroz PE, Silva RE, Aoyama AE, Lima NR. Intervenções de enfermagem e os cuidados ao idoso com doença de Parkinson. Revista Brasileira Interdisciplinar de Saúde (ReBIS). 2020;2(3):29-31.

[4] Silva PT, Carvalho ARC. Doença de Parkinson: o tratamento terapêutico ocupacional na perspectiva dos profissionais e dos idosos. Caderno Brasileira de Terapia Ocupacional. 2019;27(20):331-344. 


\section{RECISATEC - REVISTA CIENTÍFICA SAÚDE E TECNOLOGIA ISSN 2763-8405}

BENEFíCIOS DA ATIVIDADE FUNCIONAL EM IDOSOS COM DOENÇA DE PARKINSON: REVISÃO BIBLIOGRÁFICA Kauara Vilarinho, Ane Beatriz Vital de Castro, Alana Freitas Santos

[5] Agathão B. Evaluation of the quality of life of elderly people with Parkinson's disease. Revista Health Care Network. 2017;10(1).

[6] Chomiak T, Watts A, Meyer N, Pereira FV, Hu B. A training approach to improve the automaticity of stepping during the dual task in Parkinson's disease. Medicine (Baltimore). 2017;96(5):1-6.

[7] Grigoletto SEM, Brito JC, Heredia RJ. Treinamento funcional para que é para quem serve? Revista Brasileira Cineantropometria e Desempenho Humano. 2014;16(6):714-719.

[8] Poças DR, Voser CR, Junior DSAM, Aimi AG, Marques AP, Hein PA. Tratamento funcional como método de treinamento de atletas de alto rendimento. Revista Brasileira de Prescrição e Fisiologia do Exercício. 2018;12(77):694-700.

[9] Pereira MP, Costa GJ, Bezerra LI, Oliveira SL, Santos CM. Impacto do treinamento funcional no equilíbrio e funcionalidade de idosos não institucionalizados. Revista Brasileira de Ciência e Movimento. 2017;25(1):79-89.

[10] Zanardi APJ, Monteiro EP, Martins FV, Tartaruga PAL. Alterações neuromusculares em idosos com doença de Parkinson. Estudos Interdisciplinares sobre o Envelhecimento. 2019;24:177-191.

[11] Bessa RCJ. Efeitos de um programa de thai yoga massagem no equilíbrio dinâmico de indivíduos com doença de Parkinson. Brasília. Monografia [Bacharelado em Educação Física] - Universidade Federal de Brasília; 2018.

[12] Vara CA, Medeiros R, Striebel WLV. O tratamento fisioterapêutico na doença de Parkinson. Revista Neurociência. 2012:20(2):266-272.

[13] Paixão AO, Jesus FVA, Silva SF, Messias SMG, Nunes MGLT, et al. Doença de Parkinson: uma desordem neurodegenerativa. Cadernos de Graduação - Ciências Biológicas e da Saúde. 2013;1(6):57-65.

[14] Souza MFC, Almeida PCH, Sousa BJ, Silveira SSY, Bezerra LCJ. A doença de Parkinson e o processo de envelhecimento motor: uma revisão de literatura. Revista Neurociência. 2011;19(4):718723.

[15] Ferreira LL, Cavenaghi MO. Capacidade funcional em idosos com doença de Parkinson: revisão de literatura. SAÚDE REV. 2017;17(47):73-81.

[16] Nascimento BCI, Santos OCR, Guerreiro FC, Costa NCA, Camelier RWF. Avaliação postural em pessoas com doença de Parkinson. Revista Pesquisa em Fisioterapia. 2016;(1):56-64.

[17] Brito RMG, Souza GRS. Distúrbios motores relacionados ao mal de Parkinson e a dopamina. Revista UNINGÁ. 2019;56(3):95-105.

[18] Rocha GSM. Alterações posturais na doença de Parkinson. Revista Neurociência. 2015;23(4):475476.

[19] Nascimento DA. Importância do treinamento funcional para os idosos na melhora do equilíbrio. Revista Brasileira de Reabilitação e Atividade Física. 2019;8(1):21-26.

[20] Neto RGA, Grigoleto SEM, Santos SM, Cyrino SE. Treinamento funcional para idosos: uma breve revisão. Revista Brasileira de Ciência e Movimento. 2016;24(2):167-177.

[21] Fatori OC, Leite FC, Souza SPAL, Patrizzi JL. Dupla tarefa e mobilidade funcional de idosos ativos. Revista Brasileira Geriatria Gerontologia. 2015;18(1):29-37. 


\section{RECISATEC - REVISTA CIENTÍFICA SAÚDE E TECNOLOGIA ISSN 2763-8405}

BENEFíCIOS DA ATIVIDADE FUNCIONAL EM IDOSOS COM DOENCA DE PARKINSON: REVISÃO BIBLIOGRÁFICA Kauara Vilarinho, Ane Beatriz Vital de Castro, Alana Freitas Santos

[22] Ferreira PJ, Cruz LV, Cardoso AR, Leite STNF, Duarte GMC, Gouveia MV. Efeitos de treino de equilíbrio e coordenação motora em idosos: ensaio clínico randomizado. Revista Brasileira Geriatria e Gerontologia 2012.

[23] Gusmão SFM, Reis AL. Efeitos do treinamento sensório-motor no equilíbrio de idosos: revisão sistemática. Revista Saúde Coletiva UFES. 2017;7(1):64-70.

[24] Flores TF, Rossi GA, Scbmidt SP. Avaliação do equilíbrio corporal na doença de Parkinson. Arquivos Internacionais de Otorrinolaringolia. 2011;15(2):142-150.

[25] Rodrigues IRF. O profissional de educação física e suas intervenções metodológicas para melhorar a coordenação motora em idosos com Parkinson. Goiânia. Monografia [Licenciatura em Educação Física] - Pontifícia Universidade Católica de Goiás; 2020.

[26] Touguinha H, Cruz RM. Efeitos do treinamento funcional na melhora das atividades da vida diária de idosos. Saúde em Foco. 2015;260-264.

[27] Silva ORK, Mejia MPD. Os benefícios da fisioterapia no paciente com Parkinson: revisão bibliográfica. Goiânia. Monografia [Pós-Graduação em Fisioterapia Neurofuncional] - Faculdade Ávila; 2013.

[28] Ortega SJ, Oliveira LT, Oliveira VD, Benedeti RM, Bertolini GMMS. Avaliação da marcha, equilíbrio, e qualidade de vida em indivíduos com a doença de Parkinson submetidos ao tratamento por meio da hidroterapia. Revista Inspirar, Movimento e Saúde. 2014;6(4):11.

[29] Santos MD, Marques LG, Dahmer VSD, Crepaldi SLM, Crepaldi AA, Silva ML, et al. Tratamentos farmacológicos e fisioterapêuticos na melhora da qualidade de vida dos pacientes com doença de Parkinson. Revista FAIPE. 2018;8(1):60-71.

[30] Gondim OGTI, Lins ASCC, Coriolano SWGM. Exercícios terapêuticos domiciliares na doença de Parkinson: uma revisão integrativa. Revista Brasileira Geriatria Gerontologia. 2016;19(2):349-364.

[31] Terra BM, Santos SMS. Conceito Bobath e uso da bola terapêutica na melhora do equilíbrio e da marcha em indivíduos com doença de Parkinson. Revista Terra \& Cultura: Cadernos de Ensino e Pesquisa. 2017;1:49-52.

[32] Bianchetti M, Santos VM. Efeitos da hidroterapia no equilíbrio e na sensibilidade do pé em indivíduos com doença de Parkinson. Revista Destaques Acadêmicos. 2019;11(3):230-242.

[33] Gonçalves AC, Montero AG, Freitas BAN. A importância da cinesioterapia no equilíbrio do idoso. Revista discente da UNIABEU. 2016;4(8):5-10.

[34] Luna SMN, Brech CG, Canonica A, Ernandes CR, Bocalini SD, Greve AJ, Alonso CA. Efeitos do treinamento da marcha em esteira em idosos com doença de Parkinson: uma revisão da literatura. Einstein. 2020;18:1-9.

[35] Oliveira, AD, Monteiro WVLA, Pinheiro BJC. Treinamento funcional: um estudo bibliográfico sobre os conceitos e aplicações. Revista de Educação Física. 2013;82(157):12-21.

[36] Cândido PD, Cillo LAB, Fernandes AS, Nalesso PR, Jakaitis F, Santos GD. Análise dos efeitos da dupla tarefa na marcha de pacientes com doença de Parkinson. Revista Neurociência. 2012;20(2):240245.

[37] Gléria MDP, Sandoval AR. Treinamento funcional como recurso fisioterapêutico para o aprimoramento da força muscular e equilíbrio de idosos. Efdesportes.com: revista digital. 2011;16(161).

[38] Lopes RM, Carvalho OA. Os benefícios do treinamento de dupla tarefa em pessoas com doença de Parkinson. Goiânia. [Pós-Graduação em Fisioterapia Neurofuncional] - Faculdade Ávila; 2012. 


\section{RECISATEC - REVISTA CIENTÍFICA SAÚDE E TECNOLOGIA}

\section{ISSN 2763-8405}

BENEFÍCIOS DA ATIVIDADE FUNCIONAL EM IDOSOS COM DOENÇA DE PARKINSON: REVISÃO BIBLIOGRÁFICA Kauara Vilarinho, Ane Beatriz Vital de Castro, Alana Freitas Santos

[39] Fernandes SH, Tuon T, Rasado M, Lino RP, Pinho AR. Efeitos do exercício físico de dupla tarefa sobre a instabilidade postural e parâmetros respiratórios em pacientes com doença de Parkinson. Revista Brasileira Fisiologia Exercício. 2021;20(1):52-63.

[40] Almeida Al, Bueno BEM, Andrello RCA, Batistetti LC, Lemes BL, Barboza MN, et al. Fisioterapia baseada no treinamento de dupla tarefa no equilíbrio de indivíduos com doença de Parkinson. Revista Saúde (Santa Maria). 2015;41(2):71-80.

[41] Vale SL, Alves FG, Ribeiro FM. Treino de dupla tarefa no tratamento de pacientes com doença de Parkinson: revisão de literatura. Scientia Generalis. 2020;1(1):64. 